\title{
EPIDEMIOLOGIA DE 118 CASOS DE ESCLEROSE MÚLTIPLA COM SEGUIMENTO DE 15 ANOS NO CENTRO DE REFERÊNCIA DO HOSPITAL DA RESTAURAÇÃO DE PERNAMBUCO
}

\author{
Maria Lúcia Brito Ferreiraํㅡㄹ Maria Íris Moraes Machado², Maria Lúcia Vilela², \\ Maria José Guedes ${ }^{3}$, Luis Ataíde J r ${ }^{4}$, Silvana Santos4 ${ }^{4}$ Sílvia Gomes Laurentino ${ }^{4}$
}

\begin{abstract}
RESUMO - A prevalência de esclerose múltipla (EM) varia consideravelmente no mundo. De acordo com Kurtzke, a América do Sul é considerada região de baixa prevalência (menor que 5 casos:100000 habitantes). Objetivo: Descrever a epidemiologia da EM em centro de referência, comparando-a aos achados de oito serviçosnacionais. Método: Através de estudo de incidência, descritivo, prospectivo, longitudinal, foram analisados dados de 118 pacientes, atendidosno Centro de Referência para Atenção ao Paciente Portador de Doença Desmielinizante do Hospital da Restauração - Recife - PE - Brasil (CRAPPDD-HR), entre janeiro de 1987 e março de 2002, com diagnóstico de EM, segundo critérios de Poser. Resultados: Os pacientes foram acompanhados por um a 15 anos. Noventa e cinco $(80,5 \%)$ pacientes estavam em tratamento específico com imunomoduladores. A prevalência foi igual a 1,36:100000 habitantes. Identificaram-se: predomínio da etnia parda (110 casos, 93,2\%); 82 (82,2\%) casos clinicamente definidos, 15 (12,7\%) laboratorialmente definidose 6 (5,1\%) dinicamente prováveis; número máximo de surtosigual a 46; maior número de casos com EDSS leve, assim como um a 10 anos de doença na forma surto/remissão (SR). A razão de gênero dos 83 casos da forma SR se equivaleu à geral $(4,1: 1)$, contudo na forma SR com progressão secundária houve predomínio duas vezes maior para o sexo feminino e na forma primariamente progressiva, 5,5 vezes maior para o sexo masculino. Conclusão: Apesar da presente pesquisa ter se assemelhado aos oito estudos utilizados para comparação, as diferenças constatadas irão requerer novas pesquisas e novas abordagens.
\end{abstract}

PALAVRAS-CHAVE: esclerose múltipla, epidemiologia, centro de referência.

\begin{abstract}
Epidemiology of 118 cases of multiple sclerosis after 15 years of follow-up on the reference center of Hospital da Restauração, Recife, Pernambuco, Brazil

ABSTRACT - The prevalence of multiple sclerosis (MS) has a considerable variability all over the world. According to Kurtzke, South America is considered to have low prevalence (minor than 5 cases: 100000 habitants). Objective: To describe the MSepidemiology on a reference center, compared to eight Brazilian series. Method: according to a descriptive, prospective, longitudinal, prevalence study, we analyzed data of 118 patients, who attended at the Reference Center for Attention to Patients with Demielinizating Diseases of Hospital da Restauração (CRAPPDD-HR) - Recife - Pernambuco, Brazil, from January 1987 to March 2002, to have MS diagnosed according to Poser criteria. Results: The patients were followed up by 1 to 15 years. Ninety five $(80.5 \%)$ patients were in treatment with immune-modulators. The prevalence was 1,36:100000 habitants. We have identified: predominance of mulattoes (110,93.2\%), $82(82.2 \%)$ casesclinically defined, $15(12.7 \%)$ cases laboratorial defined and $6(5.1 \%)$ cases clinically probable; a number of maximum bouts equal to 46; more frequent mild EDSS for patients with 1 to 10 years of MS of remittent-recurrent (RR) form. Gender rate of 83 cases of RR form wasequivalent to the general one, however on RR with secondary progressive form form, the rate was 2.5 timesgreater for femalesthan for males, and on primary progressive form, 5.5 times greater for males. Condusion: Although some results of this research had been similar to that of eight studies used as standards for comparison, the differences will require new approaches.
\end{abstract}

KEY WORDS: multiple sclerosis, epidemiology, reference center.

Centro de Referência para Atenção ao Paciente Portador de Doença Desmielizante do Hospital da Restauração, Recife PE, Brasil (CRAPPDD-HR): ${ }^{1}$ Neurologista Chefe do Serviço de Neurologia e Coordenadora do Centro; ${ }^{2}$ Neurologistas do CRAPPDD-HR; ${ }^{3}$ Enfermeira do CRAPPDD-HR; ${ }^{4}$ Neurologista.

Recebido 20 Junho 2003, recebido na forma final 4 J unho 2004. Aceito 23 Julho 2004.

Dra. Maria Lúcia Brito Ferreira - Rua Dr. José Maria 841 - 52041-000 Recife PE - Brasil. E-mail: lucabrito@uol.com.br 
A esclerose múltipla (EM) é doença inflamatória desmielinizante do sistema nervoso central (SNC), com presumida origem auto-imune e caracterizada por infiltração multifocal de linfócitos T auto-reativos do sistema imune, através da barreira hematoencefálica ${ }^{1}$. Fatores exógenose genéticos são inequívocosna susceptibilidade e na expressão da doença por atuarem no balanço regularizador de citocinas pró-inflamatóriase anti-inflamatórias ${ }^{1}$. Apresenta variabilidade ampla de sinaise sintomas clínicos, assim como em sua história natural ${ }^{2}$.

A prevalência de EM varia consideravelmente no mundo ${ }^{2}$. A razão para essa variação prevalência/ incidência não é conhecida. De acordo com Kurtzke e Page ${ }^{3}$, a América do Sul é considerada região de baixa prevalência, com taxa menor que 5 casos por 100.000 habitantes.

Neste estudo, apresenta-se uma análise epidemiológica de desenho prospectivo, longitudinal, de 118 pacientes com diagnóstico de EM de acordo com os critérios de Poser ${ }^{4}$, acompanhados no Centro de Referência para Atenção ao Paciente Portador de Doença Desmielinizante, do Hospital da Restauração - Recife, Pernambuco (CRAPPDD-HR). Osachados são discutidose comparadosaos de outras séries nacionais.

\section{MÉTODO}

Através de estudo de incidência, descritivo, prospectivo, longitudinal, analisaram-se os dados de 118 pacientes, admitidos no centro de referência, por demanda espontânea ou por encaminhamento de neurologistas não pertencentes ao CRAPPDD-HR, no período de janeiro de 1987 a março de 2002, com indicação para acompanhamento e tratamento, oriundos de ambulatório privado $(42,4 \%)$, ambulatório público $(50,8 \%)$ ou hospital público $(6,8 \%)$. Todos os pacientesforam submetidosà rotina de revisão clínica, laboratorial geral e espećfica, estabelecida no Centro para diagnóstico primário ou confirmatório e classificação de acordo com os critérios de Poser ${ }^{4}$. A incapacidade (sistemasfuncionais) foi avaliada utilizando EDSS, como descrito por Kurtzke e Page ${ }^{3}$.

Com base nos achados dos exames clínicos, de imagem e laboratoriais específicos, os dados constantes de banco geral de registro epidemiológico e clínico foram organizadose analisados com o programa EPI-INFO versão 6.04d, da Organização Mundial de Saúde, utilizandose medidas de posição e de dispersão, distribuições de freqüências absolutas e relativas, assim como teste de qui quadrado, ao nível de significância de 0,05, para análise de contingência, respeitadosos critérios de Cochran.

\section{RESULTADOS}

Na Figura 1 estão representados os 29 municípios de origem dos pacientes atendidosno período de janeiro de 1987 a março de 2002, dos quais $82,9 \%$ situadosno Estado de Pernambuco, 3,4\% na Bahia, 3,4\% no Rio Grande do Norte, 3,4\% em Alagoas e $6,9 \%$ na Paraíba. A prevalência de EM foi igual a 1,36:100000 habitantes, tomando por base o total populacional do Estado de Pernambuco, informado pelo censo demográfico de $2000^{5}$.

Na Figura 2 estão expressosos percentuais de pacientes ingressados e dos acompanhados no CRAPPDD-HR, no período de 1987 a 2002, observando-se aumento progressivo, ao longo de 15 anos, sem nenhuma evasão e com redução de apenas dois casos por óbito não relacionado à doença de base.

Somenteum $(0,9 \%)$ paciente era negro; sete $(5,9 \%)$ eram de etnia branca e 110 (93,2\%) eram pardos.

De acordo com os critérios de Poser 4 , a distribuição dos pacientes foi: 97 (82,2\%) clinicamente definidos $\left(A_{1}\right.$ e $\left.A_{2}\right), 15(12,7 \%)$ laboratorialmente definidos e $6(5,1 \%)$ clinicamente prováveis.

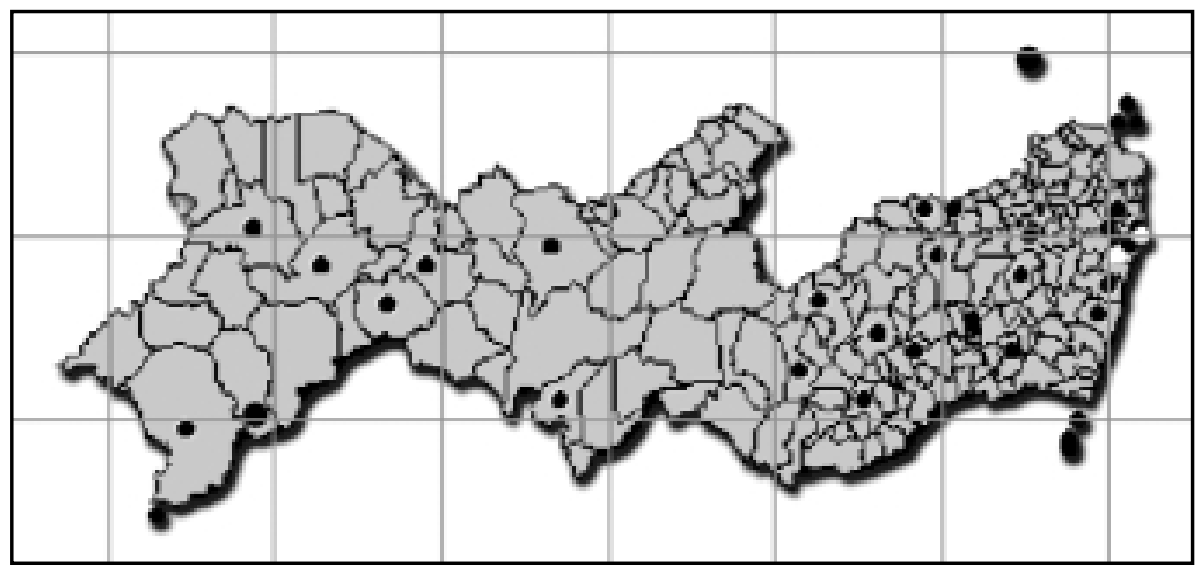

Fig 1. Distribuição geográfica dos 118 casos de EM atendidos no CRAPPDD-HR, em 28 municípios de quatro Estados: janeiro 1987-março 2002. 


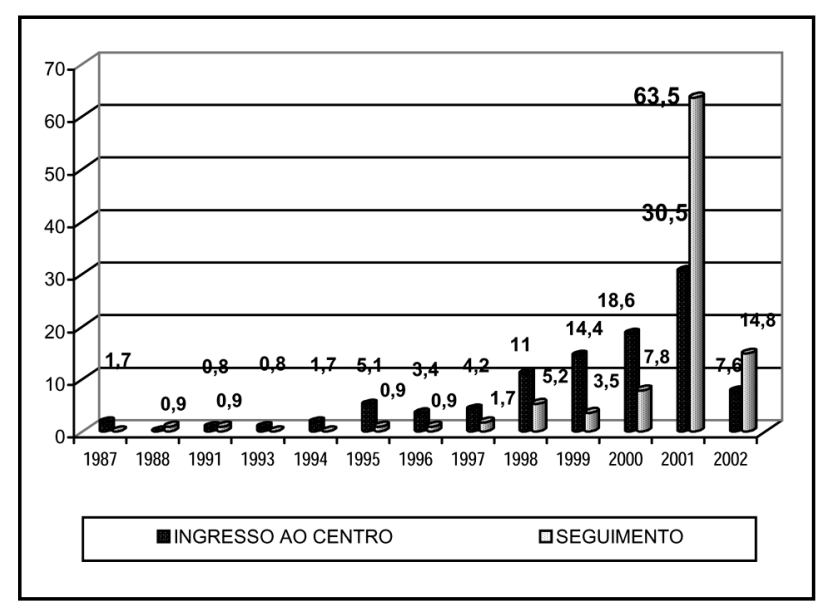

Fig 2. Distribuição de primeira consulta e de seguimento de pacientes com EM: CRAPPDD-HR, janeiro 1987-março 2002.

A apresentação clínica maisfreqüente foi a surto/remissão (SR), com 83 casos (70,4\% ), seguida pela forma clínica surto/remissão com progressão secundária (SP) (28 pacientes, 23,7\%) e 7 casos da forma primariamente progressiva (PP) (5,9\%), conforme apresentado na Tabela 1, da qual também constam a idade média de início da EM, a razão de gênero geral e segundo forma clínica. A razão de gênero dos 83 casos da forma SR se equivaleu à geral $(4,1: 1)$, contudo na forma SP houve predomínio duas vezes maior para o sexo feminino e na forma PP, 5,5 vezes maior para o sexo masculino.

O número de surtos variou de zero a 46 episódios nos grupos SR e SP. No grupo PP, com seguimento de 1 a 40 anos, o EDSS, em 5 deles, esteve entre 5,0 e 7,0 pontos, em um caso o escore foi 9,0 e em outro, igual a 3,0. Dos 83 pacientes com a forma clínica SR, o EDSS variou de 0 a 6,5, com um desvio para a esquerda, ou seja, com tendência a valores inferiores a 3,5 . Nove pacientes $(10,8 \%)$ apresentaram a forma benigna, de acordo com a definição proposta por Noseworthy et al. ${ }^{2}$ (EDSS menor que 3,5 após 10 anos de doença). Dentre os 28 pacientes com EDSS variando de 2,0 a 9,0 pontos, com a forma SP, houve desvio à direita, isto é, tendência de EDSS maiores que 5,0 (Tabela 1).

A distribuição da EDSS segundo forma clínica está expressa na Tabela 2, na qual se classificaram como forma leve a EDSSigual ou inferior a 3,5, moderada quando entre 4,0 e 6,5 e severa, superior a 6,5. Identificou-se que, para a forma SR, predominaram tempo entre um e 10 anos de doença com EDSSleve, enquanto que na forma SP detectou-se maior número de casos com 1 a 20 anos de doença e formas moderada a severa à EDSS. A pesar do número de pacientes com a forma PP ter sido reduzido, pode-se constatar predomínio das formas mais graves, mesmo para os pacientes com um a 10 anos de doença.

Asterapêuticas disponíveis, sintomática e específica, foram utilizadas em definição pactuada entre os profissionais e os pacientes, estando 95 (80,5\%) em uso de medicação e 23 (19,5\%) sem uso de drogas.

\section{DISCUSSÃO}

Os estudos epidemiológicos são como a fotografia de um momento histórico em um serviço e

Tabela 1. Distribuição dos parâmetros demográficos e de variação da EDSS segundo formas clínicas de 118 portadores de EM. CRAPPDD-HR, janeiro 1987-março 2002.

\begin{tabular}{|c|c|c|c|c|}
\hline \multirow[t]{2}{*}{ Parâmetros } & \multicolumn{3}{|c|}{ Formas Clínicas } & \multirow[t]{2}{*}{ Geral } \\
\hline & surto/remissão & $\begin{array}{l}\text { surto/remissão } \\
\text { com progressão } \\
\text { secundária }\end{array}$ & $\begin{array}{c}\text { primariamente } \\
\text { progressiva }\end{array}$ & \\
\hline \multicolumn{5}{|l|}{ Demográficos } \\
\hline Idade de início (anos) & $33,2 \pm 11,5$ & $30,3 \pm 11,6$ & $41,1 \pm 13,8$ & $39+12,5$ \\
\hline \multicolumn{5}{|l|}{ Sexo } \\
\hline feminino & $67(70,5 \%)$ & $25(26,3 \%)$ & $3(3,2 \%)$ & $95(100 \%)$ \\
\hline masculino & $16(69,6 \%)$ & $3(13 \%)$ & $4(17,4 \%)$ & $23(100 \%)$ \\
\hline Razão de gênero (F:M) & $4,2: 1$ & $8,3: 1$ & $0,75: 1$ & $4,1: 1$ \\
\hline \multicolumn{5}{|l|}{ Variação da EDSS } \\
\hline Intervalo de variação & $0-6,5$ & $2,0-9,0$ & $0-9,0$ & $0-9,0$ \\
\hline Tendência & $<3,5$ & $>5,0$ & ausente & - \\
\hline Total & $83(70,4 \%)$ & $28(23,7 \%)$ & $7(5,9 \%)$ & $118(100 \%)$ \\
\hline
\end{tabular}


Tabela 2. Distribuição de formas clínicas e tempo de doença de 118 pacientes segundo classificação pela EDSS, CRAPPDD-HR, janeiro 1987-março 2002.

\begin{tabular}{|c|c|c|c|c|c|c|}
\hline \multirow[t]{3}{*}{ Formas clínicas e tempo de doença (anos) } & \multicolumn{6}{|c|}{ Classificação pela EDSS } \\
\hline & \multicolumn{2}{|c|}{ leve } & \multicolumn{2}{|c|}{ moderada } & \multicolumn{2}{|c|}{ severa } \\
\hline & $\mathrm{n}$ & $\%$ & $\mathrm{n}$ & $\%$ & $\mathrm{n}$ & $\%$ \\
\hline \multicolumn{7}{|l|}{ surto/remissão } \\
\hline 1 a 10 & 59 & 71,1 & 8 & 9,6 & - & - \\
\hline 11 a 20 & 9 & 10,9 & 3 & 3,6 & 1 & 1,2 \\
\hline 21 a 30 & 2 & 2,4 & 1 & 1,2 & - & - \\
\hline \multicolumn{7}{|l|}{ surto/remissão com progressão secundária } \\
\hline 1 a 10 & 1 & 3,6 & 6 & 21,4 & 5 & 17,8 \\
\hline 11 a 20 & 3 & 10,7 & 3 & 10,7 & 6 & 21,4 \\
\hline 21 a 30 & 1 & 3,6 & 1 & 3,6 & 1 & 3,6 \\
\hline 41 a 50 & 1 & 3,6 & - & - & - & - \\
\hline \multicolumn{7}{|l|}{ progressão primária } \\
\hline 1 a 10 & - & - & 3 & 42,8 & 2 & 28,6 \\
\hline 11 a 20 & 1 & 14,3 & - & - & - & - \\
\hline 31 a 40 & - & - & - & - & 1 & 14,3 \\
\hline
\end{tabular}

Leve correspondeu a EDSS entre 0 e 3,5; moderada, entre 4,0 e 6,5 e severa, maior que 6,5.

têm o objetivo de permitir comparações, de forma a que sejam identificadas características gerais ou específicas de um local ${ }^{6}$. Esses diagnósticos situacionaispermitem adoção de condutas terapêuticas e diagnósticas particulares a cada caso, com meIhora da prestação dos serviços médicos. Por esse motivo, os dados desse trabalho são comparados aos de outras séries históricas, para que se comentem apenasosaspectos mais relevantes das diferenças encontradas, uma vez que o CRAPPDD-HR é serviço de referência estadual no qual há 15 anos e doismeses vêm sendo atendidos todosos pacientes com suspeita de EM, provenientes de todas as clínicas e hospitais privados, assim como de todosos ambulatóriose hospitais públicos, não restringindo sua clientela a um único hospital. Este estudo deve ser considerado prospectivo, já que todos os pacientes foram acompanhados, sistematicamente, desde o primeiro atendimento no Serviço de Neurologia, posteriormente denominado CRAPPDD-HR em 2002, daí se justificar o aumento do número de pacientesatendidos. Na Tabela 3 estão expostos osresultados de oito estudosepidemiológicosbrasileiros sobre EM, que serviram de base para avaliar os achados ${ }^{7-14}$.

A prevalência de 1,36: 100000 habitantes é baixa segundo a dassificação de Kurtzke e Page ${ }^{3}$. Callegaro ${ }^{10}$ referiu estimativa de prevalência para o Estado de São Paulo superior a 5,0\%. As causas para a prevalência do presente trabalho poderão ser alvo de novas pesquisas que abordem os aspectos raciais, socioeconômicose de localização geográfica.

A diferença identificada entre os oito estudose este, quanto à distribuição racial, pode se dever à variabilidade de critérios adotados para classificar preto, branco, pardo e mulato, o que poderia explicar a alta incidência de pretos no estudo de $\mathrm{Pa}$ paiz-Alvarenga et al. ${ }^{11}$, assim como de pardos no presente estudo, no qual considerou-se preto o indivíduo de pele negra, cabelos encaracolados, lábios grossos e com antecedentes de primeira geração pretos, assim como branco, aquele de pele branca, cabelos claros, olhos claros, tal como pais e irmãos, estando todas as demais raças sob a denominação de pardos, adotando-se a classificação de fototipos de Norris, et al. ${ }^{15}$. Embora nem todos os estudos epidemiológicos consultados (Tabela 3) tenham apresentado o percentual discriminado por critério de Poser $^{4}$, comparando os dados deste trabalho com osde Arruda et al. ${ }^{9}$, de Papaiz-Alvarenga et al. ${ }^{11}$, de Leite, Andrade e Novis ${ }^{13}$ e de Oliveira et al. ${ }^{14}$, observou-se menor percentual da forma CP (clinicamente provável), porém muito mais elevado da forma LD (laboratorialmente definida).

Quanto à razão de gênero, foi interessante identificar, não apenasmaior risco do sexo feminino comparado às razões de todos os outros trabalhos, mas ainda maior para a forma SP (secundariamente progressiva), ao passo que os indivíduos do sexo masculino apresentaram maior risco à forma PP 
Tabela 3. Resultados de nove estudos epidemiológicos brasileiros sobre EM.

\begin{tabular}{|c|c|c|c|c|c|c|}
\hline Autores & Amostra & $\begin{array}{l}\text { Razão de } \\
\text { gênero F:M }\end{array}$ & $\begin{array}{l}\text { Idade média } \\
\text { de início }\end{array}$ & Raça & $\begin{array}{l}\text { Forma } \\
\text { clínica }\end{array}$ & $\begin{array}{l}\text { Critério } \\
\text { de poser }\end{array}$ \\
\hline Moreira et al. ${ }^{7}$ & 302 & 3,$1 ; 1$ & 37,7 & $\begin{array}{c}94,0 \% \mathrm{Br} \\
5,0 \% \mathrm{P} \\
1,0 \% \mathrm{As}\end{array}$ & $\begin{array}{l}72,0 \% \text { SR } \\
14,0 \% \text { PP } \\
14,0 \% \text { SP }\end{array}$ & EM definitiva \\
\hline Tilbery et al. ${ }^{8}$ & 214 & $2,9: 1$ & $\begin{array}{l}28(\mathrm{SR}) \\
36(\mathrm{PP})\end{array}$ & $\begin{array}{c}96,0 \% \mathrm{Br} \\
4,2 \% \mathrm{P} \\
0,5 \% \mathrm{As}\end{array}$ & $\begin{array}{l}82,0 \% \text { SR } \\
18,0 \% \text { PP }\end{array}$ & EM definitiva \\
\hline Arruda et al..$^{9}$ & 200 & $1,8: 1$ & $32,0 \pm 9,9$ & $\begin{array}{c}98,5 \% \mathrm{Br} \\
1,5 \% \mathrm{P}\end{array}$ & $\begin{array}{c}91,0 \% \mathrm{SR} \\
8,0 \% \mathrm{PP} \\
1,0 \% \mathrm{SP}\end{array}$ & $\begin{array}{c}A-61,0 \% \\
B-21,0 \% \\
C-8,0 \%\end{array}$ \\
\hline Callegaro ${ }^{10}$ & 120 & $1,6: 1$ & $27,9 \pm 8,9$ & $\begin{array}{c}79,2 \% \mathrm{Br} \\
10,0 \% \mathrm{M} \\
10,0 \% \mathrm{P} \\
0,8 \% \mathrm{As}\end{array}$ & $\begin{array}{c}85,0 \% \text { SR } \\
10,2 \% \text { PP } \\
4,2 \% \text { SP }\end{array}$ & EM definitiva \\
\hline $\begin{array}{l}\text { Papaiz-Alvarenga } \\
\text { et al. }{ }^{11}\end{array}$ & 88 & $3: 1$ & $27,9 \pm 11,3$ & $\begin{array}{l}68,2 \% \mathrm{Br} \\
31,8 \% \mathrm{P}\end{array}$ & $\begin{array}{c}88,6 \% \mathrm{SR} \\
4,5 \% \mathrm{PP} \\
6,8 \% \mathrm{SP}\end{array}$ & $\begin{array}{c}A-74,0 \% \\
B-5,3 \% \\
C-17,8 \%\end{array}$ \\
\hline $\begin{array}{l}\text { Lana-Peixoto, } \\
\text { Lana-Peixoto }^{12}\end{array}$ & 67 & $2,3: 1$ & $28,9 \pm 10,4$ & $\begin{array}{c}76,0 \% \mathrm{Br} \\
19,4 \% \mathrm{M} \\
4,5 \% \mathrm{P}\end{array}$ & - & EM definitiva \\
\hline $\begin{array}{l}\text { Leite, Andrade, } \\
\text { Novis }^{13}\end{array}$ & 51 & $2,1: 1$ & $34,5 \pm 13,9$ & $\begin{array}{c}\text { 62,7\% Br } \\
37,4 \% \text { não } \mathrm{Br}\end{array}$ & $\begin{array}{c}\text { 47,0\% SR } \\
\text { 19,7\% PP } \\
33,3 \% \text { mista }\end{array}$ & $\begin{array}{c}45 \text { EM definitiva } \\
6 \text { provável }\end{array}$ \\
\hline Oliveira et al. ${ }^{14}$ & 50 & $2: 1$ & $32,5 \pm 9,6$ & $\begin{array}{c}64,0 \% \mathrm{Br} \\
34,0 \% \mathrm{M}+\mathrm{Br} \\
2,0 \% \mathrm{As}\end{array}$ & $\begin{array}{l}60,0 \% \text { SR } \\
30,0 \% \text { PP } \\
10,0 \% \text { SP }\end{array}$ & $\begin{array}{l}76,0 \% \text { definitiva } \\
24,0 \% \text { provável }\end{array}$ \\
\hline Brito Ferreira et al. & 118 & $4,1: 1$ & $\begin{array}{l}33,2 \pm 11,5 \text { (SR) } \\
41,1 \pm 13,8 \text { (PP) } \\
30,3 \pm 11,6 \text { (SP) }\end{array}$ & $\begin{array}{c}5,9 \% \mathrm{Br} \\
0,8 \% \mathrm{P} \\
93,2 \% \mathrm{Pd}\end{array}$ & $\begin{array}{c}70,4 \% \text { SR } \\
5,9 \% \text { PP } \\
23,7 \% \text { SP }\end{array}$ & $\begin{array}{c}82,2 \% \text { CD } \\
5,1 \% \text { CP } \\
12,7 \% \text { LD }\end{array}$ \\
\hline
\end{tabular}

SR, forma dínica surto/remissão; $P$ P, forma dínica primariamente progressiva; SP,forma dínica secundariamente progressiva; $B r$, branco; $P$, preto; $M$, mulato; $A$ s, asiático; $\mathrm{Pd}$, pardo; EM, esclerose múltipla; CD ou A, clinicamente definida; LD ou B, laboratorialmente definida; $C P$ ou $C$, dinicamente provável.

(primariamente progressiva), tal como referido por Thompson et al. ${ }^{16}$.

A incidência de formas benignas, definidas segundo Noseworthy et al. ${ }^{2}$, assemelhou-se à referida por Arruda et al. ${ }^{9}$, igual a 7,0\%, significando que, mesmo no curso longo de doença, esses pacientes manterão boa qualidade de vida em virtude de baixas perdas devidas à $\mathrm{EM}^{17}$.

Embora não se tenha encontrado correlação entre EDSS, tempo de doença e número de surtos $\left(\chi^{2}=0,67 ; g . I .=1 ; p=0,441\right)$, refletindo a dificuldade de prever o prognóstico do paciente, a identificação das tendências das formas surto/remissão e surto/remissão com progressão secundária, respectivamente para a esquerda (valores da EDSS inferio- resa 3,5) e para a direita (valores da EDSS superiores a 5,0), é importante, pois pode sugerir um mecanismo próprio ainda não definido.

Embora a literatura pertinente recomende a instituição precoce do tratamento para reduzir o aparecimento de surtos e a progressão da doença, no Centro de Referência, essa decisão é tomada pelo paciente, a partir das informações a ele prestadas pelo neurologista e equipe, em linguagem acessível, clara e tecnicamente atualizada. Por esse motivo, 23 pacientes mantiveram-se sem uso de drogas.

\section{REFERÊNCIAS}

1. Comi G, Filippi M, Wolinsky JS. European/Canadian Multicenter, double-blind, randomized, placebo-controlled study of the effects of glatiramer acetate on magnetic resonance imaging-measured disease 
activity and burden in patients with relapsing multiple sclerosis. Ann Neurol 2001;49:290-297.

2. Noseworthy JH, Lucchinetti C, Rodriguez M, Weinshenker BG. Multiple sclerosis. N Engl J Med 2000;343:938-951.

3. Kurtzke JF, Page WF. Epidemiology of multiple sclerosis in US veterans: VII. Risk factors for MS. Neurology 1997;48:204-213.

4. Poser CM. Multiple sclerosis: diagnosis and treatment. Med Principles Pract 1992;93:16.

5. Brasil. Instituto Brasileiro de Geografia e Estatística - IBGE. Vou te contar - Revista do Censo 2000. Centro de Documentação e Disseminação de Informações 2002;10:6-10.

6. Rothman KJ. Epidemiologia moderna. Sevilla:Dias dos Santos, 1997:5-41.

7. Moreira MA, Felipe E, Mendes MF, Tilbery CP. Esclerose múltipla: estudo descritivo de suas formas clínicas em 302 casos. Arq Neuropsiquiatr 2000;58:460-466.

8. Tilbery CP, Felipe E, Moreira MA, Mendes MF, França AS. Interferon beta 1a na esclerose múltipla: experiência de um ano em 62 pacientes. Arq Neuropsiquiatr 2000;58:452-459.

9. Arruda WO, Scola RH, Teive HAG, Werneck LC. Multiple sclerosis: report on 200 cases from Curitiba, Southern Brazil and comparison with other Brazilian series. Arq Neuropsiquiatr 2001;59:165-170.
10. Callegaro D. Contribuição ao estudo clínico evolutivo da esclerose múltipla: análise de 120 pacientes. Tese, Universidade de São Paulo. São Paulo, 1989.

11. Papais-Alvarenga RM, Santos CMM, Abreu JS, et al. Esclerose múltipla:perfil clínico e evolutivo no município do Rio de Janeiro. Rev Bras Neurol 1995;31:75-87.

12. Lana-Peixoto M, Lana-Peixoto MIV. Is multiple sclerosis in Brazil and Asia alike? Arq Neuropsiquiatr 1992;50:119-125.

13. Leite ACCB, Andrade C, Novis S. Esclerose múltipla no Rio de Janeiro: apresentação clínica em 51 casos. Arq Neuropsiquiatr 1990;48(Supl):66A.

14. Oliveira EML, Annes M, Oliveira ASB, Gabbai AA. Esclerose múltipla: estudo clínico de 50 pacientes acompanhados no Ambulatório de Neurologia UNIFESP-EPM. Arq Neuropsiquiatr 1999;57:51-55.

15. Norris PG, Gange RW, Hawk JL. Acute effects of ultraviolet radiation on the skin. In Fitzpatrick TB, Freedberg IM, Eisen AZ, Katz SI, Wolff K, Goldsmith LA, Austen KF, Goldsmith L (eds). Dermatology in general medicine. 4. Ed. New York: McGraw-Hill; 1993:1651-1658.

16. Thompson AJ, Polman $\mathrm{CH}$, Miller $\mathrm{DH}$, et al. Primary progressive multiple sclerosis. Brain 1997;120:1085-1096.

17. Lyseng-Williamsone KA, Plosker GL. Tratamento da esclerose múltipla remitente recorrente: definição do papel do interferon- $\beta-1^{\mathrm{a}}$ recombinante $\left(\right.$ Rebif $\left.^{\circledR}\right)$ via subcutânea. Dis Manage Health Outcomes 2002;10:307-325. 